\title{
Perirenal fat stranding is not a powerful diagnostic tool for acute pyelonephritis
}

This article was published in the following Dove Press journal:

International Journal of General Medicine

8 May 2017

Number of times this article has been viewed

\section{Hirotaka Fukami \\ Yoichi Takeuchi \\ Saeko Kagaya \\ Yoshie Ojima \\ Ayako Saito \\ Hiroyuki Sato \\ Ken Matsuda \\ Tasuku Nagasawa}

Department of Nephrology, Japanese Red Cross Ishinomaki Hospital, Ishinomaki, Miyagi Prefecture, Japan
Correspondence: Hirotaka Fukami Department of Nephrology, Japanese Red Cross Ishinomaki Hospital, 7I

Nishimichisita Hebita Ishinomaki, Miyagi

Prefecture 986-8522, Japan

$\mathrm{Tel}+81225217220$

Fax +81225960122

Email hiro.fuka05@gmail.com
Purpose: Pyelonephritis, an upper urinary tract infection, is a serious infection that often requires hospitalization. However, the accurate diagnosis of acute pyelonephritis can be difficult, especially among older individuals who can present with unusual symptoms. Imaging with computed tomography (CT) is not unusual in the diagnosis of pyelonephritis, with some clinicians regarding perirenal fat stranding (PFS) as a characteristic finding. However, the sensitivity and specificity of PFS in diagnosing pyelonephritis are currently unknown. We therefore sought to clarify the relevance of PFS in diagnosing acute pyelonephritis.

Patients and methods: We conducted a case-controlled retrospective analysis of medical records. The pyelonephritis group included 89 patients who had been diagnosed with acute pyelonephritis, while the control group included 319 patients who had undergone percutaneous renal biopsy. CT findings were available for both groups. The frequency of PFS and its sensitivity and specificity for the diagnosis of acute pyelonephritis were investigated.

Results: The mean ages of the pyelonephritis and control groups were $74 \pm 15$ years and $63 \pm 16$ years, respectively. A total of $28 \%$ of men were in the pyelonephritis group vs $61 \%$ of men in the control group. The frequency of PFS was $72 \%$ in the pyelonephritis group vs $39 \%$ in the control group. Age and renal dysfunction were associated with an increased frequency of PFS. After adjusting for age, sex, and renal function using a propensity score analysis, the sensitivity, specificity, and positive likelihood ratio of PFS for diagnosing acute pyelonephritis were $72 \%, 58 \%$, and 1.7 , respectively.

Conclusion: The presence of PFS was not useful in diagnosing acute pyelonephritis.

Keywords: acute pyelonephritis, perirenal fat stranding, sensitivity, specificity, urinary tract infection, CT

\section{Plain language summary}

Pyelonephritis is a serious infection of the upper urinary tract (the kidneys and ureters) that often requires hospitalization. Patients with pyelonephritis often have chills that start suddenly, fever, and pain between the ribs and hips on one or both sides of the body. Based on these symptoms, doctors may test the patient's blood or urine to diagnose pyelonephritis. Computed tomography (CT), which allows doctors to look inside the body, is useful for letting doctors determine whether something is blocking the urinary tract. This type of imaging is often used for diagnosing pyelonephritis; some doctors believe that a condition called perirenal fat stranding (PFS), in which swelling of the fat around the kidneys is visualized on CT, is a characteristic finding of pyelonephritis. However, it is not clear whether this belief is true. We thus performed this study to determine whether PFS is indeed associated with pyelonephritis. We found that PFS was not specific to pyelonephritis, ie, it could be associated with other conditions. Furthermore, other tests were more specific for pyelonephritis. We also found that CT was not the most cost-effective 
way to diagnose pyelonephritis, as patients' symptoms and laboratory parameters were more relevant diagnostic tools. We believe that our study is important as it evaluated PFS with respect to pyelonephritis.

\section{Introduction}

Urinary tract infections (UTIs) are very common. In the US, they account for $\sim 8.1$ million visits to health care providers annually. ${ }^{1}$ UTIs are termed differently depending on the part of the body affected; an infection of the kidney/upper urinary tract is called pyelonephritis, while an infection of the urethra is called urethritis. Among these infections, pyelonephritis can be complicated by sepsis, and in many cases, hospitalization is necessary for treatment. ${ }^{2}$

Classical symptoms of pyelonephritis include an abrupt onset of chills, fever, and unilateral or bilateral flank pain with costovertebral angle (CVA) tenderness. ${ }^{3}$ In addition to these physical findings, a combination of urine analysis (via microscopy and/or reagent strip), urine cultures, and blood cultures may be used to diagnose pyelonephritis. However, the diagnosis of acute pyelonephritis can be difficult for clinicians, especially among elderly patients who present with unusual symptoms, such as abdominal pain, vomiting, and diarrhea.

Computed tomography (CT) is a useful diagnostic tool for providing comprehensive anatomical and physiological information that accurately characterizes both intra- and extrarenal pathological conditions. ${ }^{3}$ Imaging with CT is not unusual in the diagnosis of acute pyelonephritis and is often used for patients who do not respond to antibiotics within 72 hours or patients with underlying conditions, such as pregnancy, diabetes mellitus, and immunocompromised state, among others. ${ }^{4,5}$ Swelling of the kidney, hypoenhanced renal parenchyma, and pronounced perirenal fat stranding (PFS) are some of the CT findings that may accompany acute pyelonephritis. ${ }^{6,7} \mathrm{Yu}$ et $\mathrm{al}^{8}$ have reported that perinephric fat stranding was found in $29.1 \%$ of patients with acute pyelonephritis.

Some clinicians regard PFS as a characteristic finding of pyelonephritis (Figure 1). ${ }^{9}$ However, to the best of our knowledge, no study has reported on the factors that are associated with PFS as well as the sensitivity and/or specificity of PFS in diagnosing acute pyelonephritis.

Thus, we aimed to evaluate the correlation between age, renal function, and frequency of PFS.

We also evaluated the effectiveness of PFS in diagnosing acute pyelonephritis.

\section{Patients and methods Study design}

This retrospective observational study was conducted at the Japanese Red Cross Ishinomaki Hospital, Ishinomaki, Miyagi,

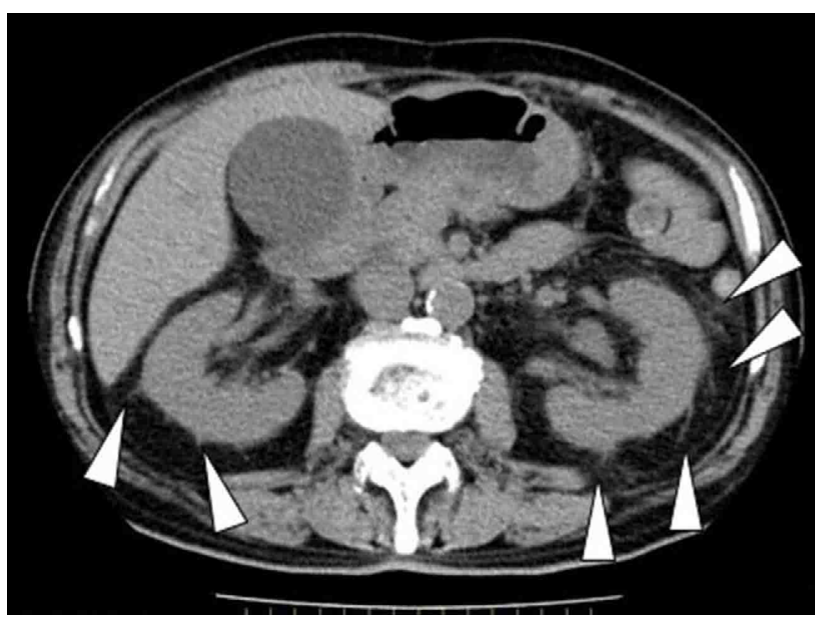

Figure I Representative image of PFS (open arrow heads). Abbreviation: PFS, perirenal fat stranding.

Japan. This study was approved by the ethical committee of Japanese Red Cross Ishinomaki Hospital (approval no 16-28) and was conducted in accordance with the 1964 Declaration of Helsinki and its later amendments or comparable ethical standards. The ethics committee deemed written informed consent not necessary due to the retrospective nature of the study.

\section{Data selection}

Patients with acute pyelonephritis were selected according to the flowchart shown in Figure 2. The medical records of all patients who were admitted to the Japanese Red Cross Ishinomaki Hospital in the Miyagi Prefecture of Japan between December 2013 and July 2015 were reviewed. Of 468,624 patients, we selected 120 patients with the 10th revision of the International Statistical Classification of Diseases and Related Health Problems (ICD-10) code N10 in their medical records.

The diagnosis of acute pyelonephritis was based on physical examination (presence of fever, chills, and CVA tenderness) and laboratory findings (increased white blood cell [WBC] count, elevated C-reactive protein [CRP], and demonstration of pyuria and bacteriuria on urine testing).

Diagnosis eligibility was assessed by internists or urologists of our hospital. We excluded eleven cases of children (aged $<16$ years) and two cases that did not demonstrate clear findings of acute pyelonephritis. Of the remaining cases, 89 had undergone CT evaluation of both kidneys; we evaluated these patients for the presence or absence of PFS as the pyelonephritis group.

We also enrolled 319 consecutive patients into the control group. These patients underwent percutaneous renal biopsy at our hospital between January 2013 and May 2016; each of them had undergone CT examination the day after the renal biopsy to check for bleeding complications. ${ }^{10}$ We had 


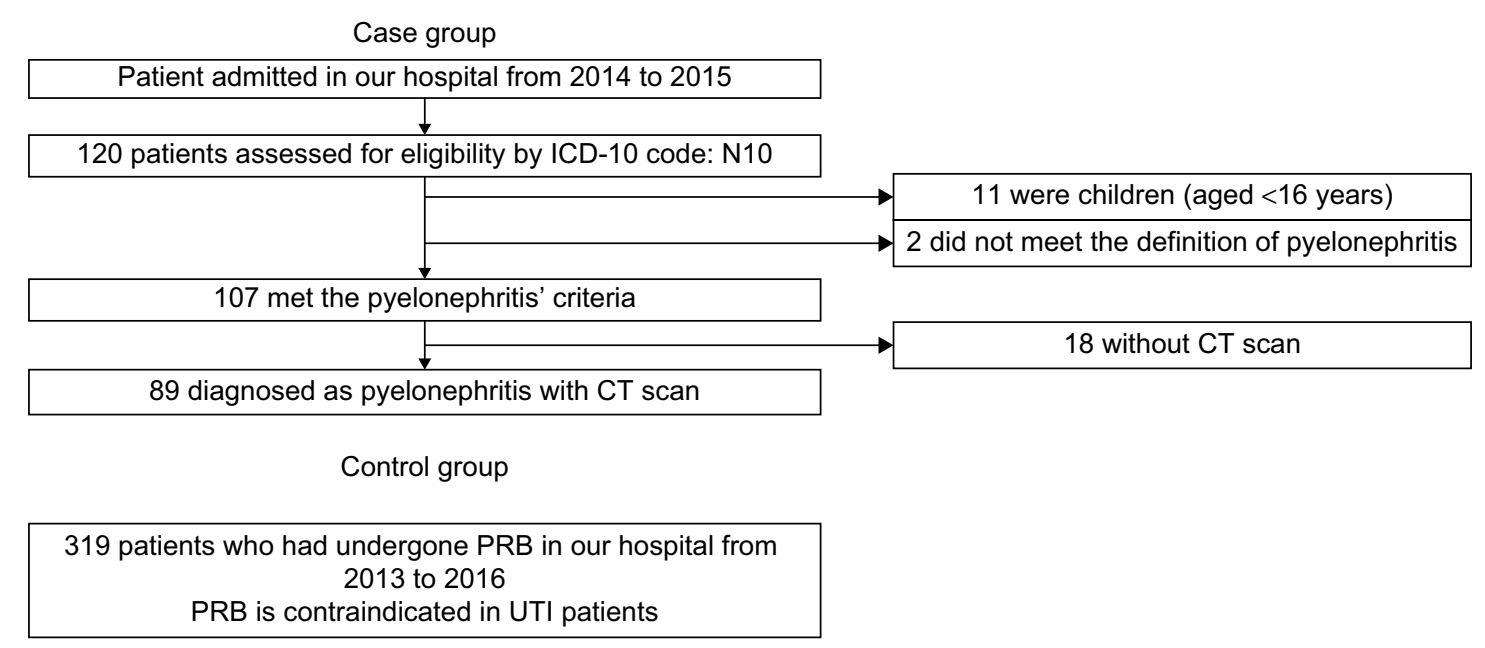

Figure 2 Flowchart showing participant screening for this study.

Abbreviations: ICD-10, I0th revision of the International Statistical Classification of Diseases and Related Health Problems; CT, computed tomography; PRB, percutaneous renal biopsy; UTI, urinary tract infection.

ruled out acute pyelonephritis prior to percutaneous renal biopsy in this group, given that acute pyelonephritis is a contraindication for renal biopsy. In this control group, PFS was evaluated on the kidney from which renal biopsy was not done. CT images were obtained using an Aquilion CXL system (Toshiba Medical Systems Corp., Tochigi, Japan).

\section{Statistical analysis}

Normally distributed data are presented as mean \pm standard deviation (SD), with nonparametric data presented as the median and 25th-75th percentile. Statistical analyses were performed using the Student's $t$-test for parametric data, the Mann-Whitney $U$ test for nonparametric data, Fisher's exact test for nominal categorical valuables, and logistic regression analysis for the following covariates: age, sex, and estimated glomerular filtration rate (eGFR).

We performed 1:1 matching based on the propensity score, as patient characteristics of both the pyelonephritis and control groups were different. ${ }^{11,12}$ The purpose of using the propensity score was to reduce the influence of bias by aligning the backgrounds of both groups. In this study, logistic regression was performed with the presence or absence of pyelonephritis as a dependent variable, and age, sex, and renal function as independent variables. A propensity score for whether or not pyelonephritis develops from a background factor was prepared. The frequency selection method was used as a forced-choice method. $p$-values of $<0.05$ were regarded as significant. All statistical data were analyzed using EZR version 3.3.1 (Saitama Medical Center, Jichi Medical University, Saitama, Japan), which is a graphical user interface for R (The R Foundation for Statistical Computing, Vienna, Austria). ${ }^{13}$

\section{Results}

The characteristics of the enrolled patients are presented in Table 1. Compared with patients in the control group, patients in the pyelonephritis group were significantly older ( $74 \pm 15$ years vs $63 \pm 16$ years, respectively, $p<0.001$ ) and had a significantly higher frequency of PFS (72\% vs $39 \%$, respectively, $p<0.01)$. There were no significant differences between both groups in terms of eGFR on admission. Other findings are as follows: 25 (28\%) and eight (9.1\%) patients in the pyelonephritis group had underlying diabetes and a history of immunosuppressant use compared to 195 (61\%) and $62(20 \%)$ patients, respectively, in the control group. A total of $26(52 \%)$ patients in the pyelonephritis group had CVA tenderness. In terms of serological findings, the median $\mathrm{WBC}$ count was $11,300 / \mu \mathrm{L}$ (interquartile range (IQR): $8,100-17,600 / \mu \mathrm{L})$ in the pyelonephritis group vs 6,100/ $\mu \mathrm{L}$ (IQR: $4,100-7,700 / \mu \mathrm{L}$ ) in the control group, while the median CRP was $12 \mathrm{mg} / \mathrm{dL}$ (IQR: $4.7-18 \mathrm{mg} / \mathrm{dL}$ ) in the pyelonephritis group vs $0.13 \mathrm{mg} / \mathrm{dL}$ (IQR: $0.05-0.56$ $\mathrm{mg} / \mathrm{dL}$ ) in the control group. The median lactate level was 1.6 in the pyelonephritis group (lactate was not measured in the control group). In terms of urinary findings, the positive rate of $\mathrm{WBC}$ was $83 \%(70 / 85)$ in the pyelonephritis group vs $20 \%(63 / 318)$ in the control group, that of nitrite was $37 \%(31 / 85)$ in the pyelonephritis group vs $4.4 \%(14 / 318)$ in the control group, and that of WBC or/and nitrite was $93 \%(79 / 85)$ in the pyelonephritis group vs $21 \%(67 / 318)$ in the control group. Bacteria were identified in urine cultures in $92 \%(71 / 77)$ of patients in the pyelonephritis group. CT imaging detected urinary stones in 40\% (36/89) of patients in the pyelonephritis group vs $12 \%(39 / 319)$ of patients in the control group, hydronephrosis in $47 \%(42 / 89)$ of patients 
Table I Patient characteristics

\begin{tabular}{|c|c|c|c|}
\hline & Case (pyelonephritis) & Control (PRB) & p-value \\
\hline Number of patients & 89 & 319 & \\
\hline \multicolumn{4}{|l|}{ Background information } \\
\hline Age (years) & $74 \pm 15$ & $63 \pm 16$ & $<0.01$ \\
\hline Male sex (\%) & $25(28 \%)$ & $195(61 \%)$ & $<0.01$ \\
\hline eGFR $\left(\mathrm{mL} / \mathrm{min} / \mathrm{l} .73 \mathrm{~m}^{2}\right)$ on admission & $34(23,54)$ & $35(23,57)$ & 0.923 \\
\hline History of diabetes mellitus & $25(28 \%)$ & $110(35 \%)$ & $<0.01$ \\
\hline History of immunosuppressant use & $8(9.1 \%)$ & $62(20 \%)$ & $<0.01$ \\
\hline CVA tapping pain & $29 / 56(52 \%)$ & - & - \\
\hline \multicolumn{4}{|l|}{ Serological findings } \\
\hline WBC count $(/ \mu \mathrm{L})$ & $11,300(8,100,17,600)$ & $6100(4,100,7,700)$ & $<0.01$ \\
\hline $\mathrm{CRP}(\mathrm{mg} / \mathrm{dL})$ & $12(4.7,18)$ & $0.13(0.05,0.56)$ & $<0.01$ \\
\hline Lactate (mmol/L) & $1.6(I . I, 2.8)$ & - & - \\
\hline \multicolumn{4}{|l|}{ Urinary findings } \\
\hline WBC & $70 / 85$ (82\%) & $61 / 318(20 \%)$ & $<0.01$ \\
\hline Nitrous acid & $3 \mathrm{I} / 85(36 \%)$ & $14 / 3 \mid 8(4.4 \%)$ & $<0.01$ \\
\hline WBC and/or nitrous acid & $79 / 85$ (93\%) & $67 / 318(21 \%)$ & $<0.01$ \\
\hline Urine culture & $71 / 83$ (92\%) & - & - \\
\hline \multicolumn{4}{|l|}{$C T$ findings } \\
\hline Urinary tract stones & $53(60 \%)$ & $39(12 \%)$ & $<0.01$ \\
\hline Hydronephrosis & $42(47 \%)$ & $12(3.8 \%)$ & $<0.01$ \\
\hline Emphysema & $3(3.4 \%)$ & I (0.3 I\%) & $<0.01$ \\
\hline PFS & 64 (72\%) & 93 (39\%) & $<0.01$ \\
\hline
\end{tabular}

Note: Data are presented as mean $\pm S D$, number (percentage), or median (interquartile range).

Abbreviations: PRB, percutaneous renal biopsy; eGFR, estimated glomerular filtration rate; CVA, costovertebral angle; WBC, white blood cell; CRP, C-reactive protein; $\mathrm{CT}$, computed tomography; PFS, perirenal fat stranding; SD, standard deviation.

in the pyelonephritis group vs $3.8 \%(12 / 319)$ of patients in the control group, and renal emphysema in $3.4 \%(3 / 89)$ of patients in the pyelonephritis group vs $0.31 \%(1 / 319)$ of patients in the control group. Among the 89 patients in the pyelonephritis group, 51 were subjected to contrast CT; of these, $22(43 \%)$ had areas where renal contrast was insufficient. All the participants in the control group underwent CT examination without contrast material.

For all enrolled patients, the sensitivity, specificity, and positive likelihood ratio of PFS in diagnosing acute pyelonephritis were $72 \%$ (95\% confidence interval [CI], 61-81), 71\% (95\% CI, 66-76), and 2.5 (95\% CI, 2.0-3.1), respectively (Table 2).

Based on our empirical speculation that PFS was affected by age, sex, and renal function, we divided patients into two groups for each of the variables: age (younger vs older), sex (male vs female), and renal function (high vs low); the frequency of PFS was determined for each group (Figures 3-5). In the pyelonephritis group, the frequency of PFS was $73 \%$ in younger patients (aged $<70$ years) vs $73 \%$ in older patients (aged $\geq 70$ years), $84 \%$ in men vs $67 \%$ in women, and $80 \%$ in those with eGFR $<35 \mathrm{~mL} / \mathrm{min} / 1.73 \mathrm{~m}^{2}$ vs $64 \%$ in those with eGFR $>35 \mathrm{~mL} / \mathrm{min} / 1.73 \mathrm{~m}^{2}$. No significant differences were found between any of these groups.
Table 2 Sensitivity, specificity, and positive likelihood ratio of PFS to diagnose acute pyelonephritis for all enrolled patients

\begin{tabular}{lllll}
\hline & & Case & Control & \\
\hline PFS & + & 64 & 93 & 157 \\
& - & 25 & 226 & 251 \\
& & 89 & 319 & 408
\end{tabular}

Note: Sensitivity, $72 \%(95 \% \mathrm{Cl}, 6 \mathrm{I}-8 \mathrm{I})$; specificity, $71 \%(95 \% \mathrm{Cl}, 66-76)$; and positive likelihood ratio, 2.5 ( $95 \% \mathrm{Cl}, 2.0-3.1)$.

Abbreviations: $\mathrm{PFS}$, perirenal fat stranding; $\mathrm{Cl}$, confidence interval.

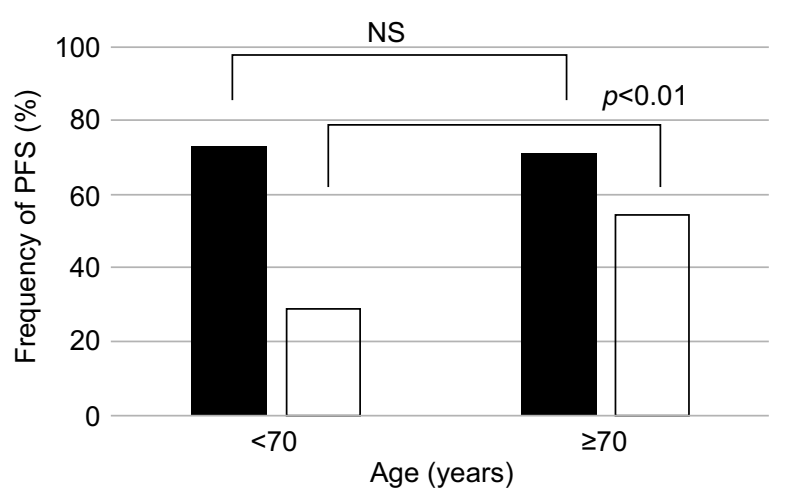

Case group $\square$ Control group

Figure 3 Frequency of PFS by age group.

Abbreviations: PFS, perirenal fat stranding; NS, not significant. 


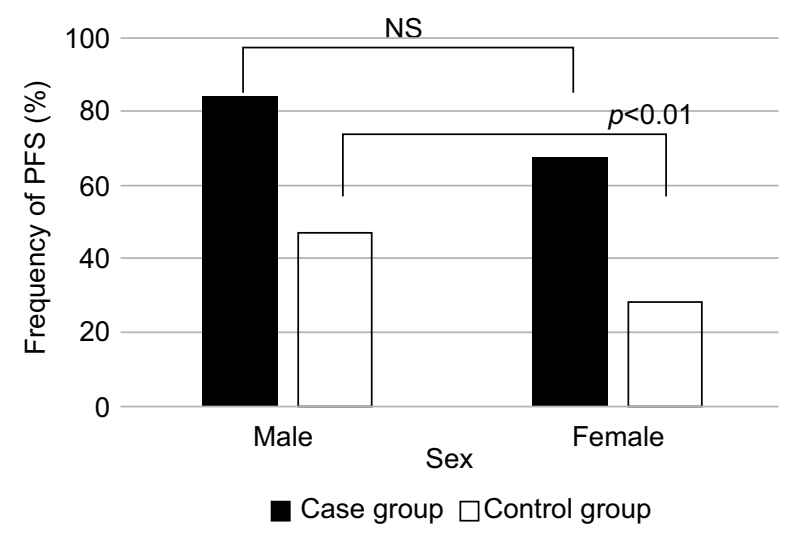

Figure 4 Frequency of PFS by sex.

Abbreviations: PFS, perirenal fat stranding; NS, not significant.

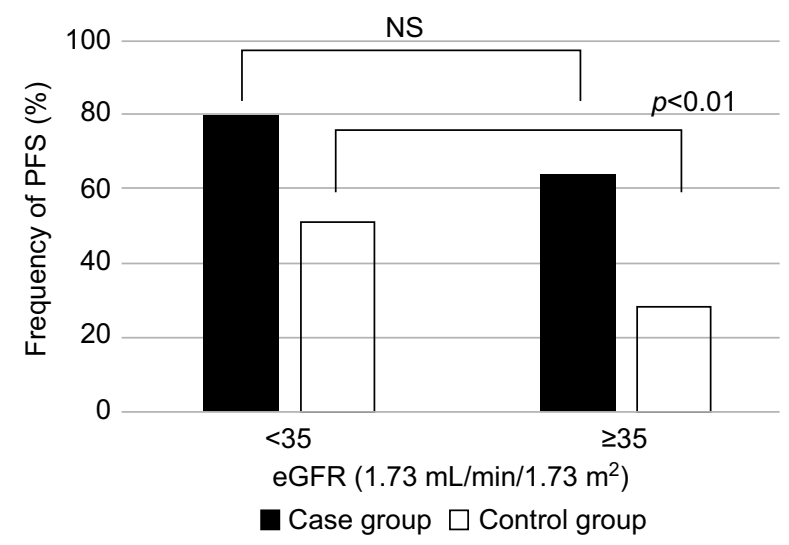

Figure 5 Frequency of PFS by eGFR.

Abbreviations: PFS, perirenal fat stranding; eGFR, estimated glomerular filtration rate; NS, not significant.

In the control group, the frequency of PFS was $29 \%$ in younger patients vs $54 \%$ in older patients, $47 \%$ in men vs $28 \%$ in women, and $51 \%$ in those with eGFR $<35 \mathrm{~mL} / \mathrm{min} / 1.73 \mathrm{~m}^{2}$ vs $28 \%$ in those with eGFR $>35 \mathrm{~mL} / \mathrm{min} / 1.73 \mathrm{~m}^{2}$.

The reason for dividing eGFR into more or less than $35 \mathrm{~mL} / \mathrm{min} / 1.73 \mathrm{~m}^{2}$ was because the median value of both the pyelonephritis and control groups was $\sim 35 \mathrm{~mL} / \mathrm{min} / 1.73 \mathrm{~m}^{2}$. The Kidney Disease: Improving Global Outcomes guidelines classify the severity of chronic kidney disease by GFR. ${ }^{14}$ GFR $30-44$ is classified as G3b category (moderately to severely decreased), and GFR 15-30 is classified as G4 category (severely decreased). Therefore, the 35 cutoff values used in this study were considered to be reasonable in general, with no significant divergence from the severity classification by GFR. Significant differences were found for each group comparison $(p<0.01)$.

A multiple logistic regression model yielding odds ratios (ORs) and 95\% CIs was then used to identify predictors of pyelonephritis. The model included age, sex, and eGFR. In the multiple logistic regression model, the OR for PFS to non-PFS was 5.0 (95\% CI, 2.7-9.3; $p<0.01)$.
Next, we performed a post hoc 1:1 person match by assigning the two groups (pyelonephritis group and control group) to each case, matched for age, sex, and eGFR on admission (Table 3 ). Conditional logistic regression analyses for these propensity score-matched data sets were used to estimate the ORs and respective $95 \%$ CIs. $^{7}$ The OR for PFS to non-PFS was 3.6 (95\% CI, $1.9-6.7 ; p<0.01)$.

After adjusting for age, sex, and renal function using the propensity score analysis, the sensitivity, specificity, and positive likelihood ratio of PFS for diagnosing acute pyelonephritis was $72 \%$ (95\% CI, 61-81), 58\% (95\% CI, 48-69), 1.7 (95\% CI, 1.3-2.3), respectively (Table 4). Using the Mantel-Haenszel test, the OR for PFS to non-PFS was found to be 2.9 (95\% CI, 1.6-6.5; $p<0.01)$.

Table 5 lists the frequency of pyogenic bacteria in urine cultures.

\section{Discussion}

This study revealed that PFS is affected by age, sex, and eGFR, with increasing age, male sex, and lower eGFR increasing the frequency of PFS. However, the presence of PFS itself was not a useful indicator of acute pyelonephritis. As shown in Table 1, PFS was found in 39\% of patients, which seemed high compared to $29.1 \%$ reported by $\mathrm{Yu}$ et al. However, in their study, the mean age of enrolled patients was $60 \pm 17$ years and $93 \%$ were females. ${ }^{8}$ In contrast, the

Table 3 Patient characteristics adjusted by age, sex, and renal function using the propensity score analysis

\begin{tabular}{llll}
\hline & $\begin{array}{l}\text { Case } \\
\text { (pyelonephritis) }\end{array}$ & $\begin{array}{l}\text { Control } \\
\text { (PRB) }\end{array}$ & p-value \\
\hline $\begin{array}{l}\text { Number of patients } \\
\text { Background information }\end{array}$ & 89 & 89 & \\
$\quad$ Age (years) & $74 \pm 15$ & $74 \pm 11$ & 0.97 \\
$\quad \begin{array}{l}\text { Male sex (\%) } \\
\left.\text { eGFR (mL/min/l.73 } \mathrm{m}^{2}\right)\end{array}$ & $25(28 \%)$ & $27(30 \%)$ & 0.87 \\
$\begin{array}{l}\text { on admission } \\
\quad \text { PFS }\end{array}$ & $64(72 \%)$ & $32(21,48)$ & 0.172 \\
\hline
\end{tabular}

Note: Data are presented as mean $\pm S D$, number (percentage), or median (interquartile range)

Abbreviations: PRB, percutaneous renal biopsy; eGFR, estimated glomerular filtration rate; PFS, perirenal fat stranding; SD, standard deviation.

Table 4 Sensitivity, specificity, and positive likelihood ratio of PFS to diagnose acute pyelonephritis after propensity score adjustment for age, sex, and renal function

\begin{tabular}{lllll}
\hline & & Case & Control & \\
\hline PFS & + & 64 & 37 & 101 \\
& - & 25 & 52 & 77 \\
& & 89 & 89 & 178 \\
\hline
\end{tabular}

Note: Sensitivity, $72 \%(95 \% \mathrm{Cl}, 6 \mathrm{I}-8 \mathrm{I})$; specificity, $58 \%(95 \% \mathrm{Cl}, 48-69)$; and positive likelihood ratio, I.7 ( $95 \% \mathrm{Cl}, \mathrm{I} .3-2.3)$.

Abbreviations: PFS, perirenal fat stranding; $\mathrm{Cl}$, confidence interval. 
Table 5 Frequency of causative bacteria

\begin{tabular}{ll}
\hline Name & Frequency, n (\%) \\
\hline Escherichia coli & $46(55 \%)$ \\
$\quad$ ESBL-producing bacteria & $4 / 46$ \\
Enterococcus faecalis & $7(8.3 \%)$ \\
Proteus mirabilis & $5(6.0 \%)$ \\
$\quad$ ESBL-producing bacteria & $2 / 5$ \\
Streptococcus sp. & $5(6.0 \%)$ \\
Staphylococcus aureus & $2 / 5$ \\
CNS & $1 / 5$ \\
Streptococcus sp. & $5(6.0 \%)$ \\
Streptococcus agalactiae & $2 / 5$ \\
Corynebacterium sp. & $4(4.8 \%)$ \\
Klebsiella pneumoniae & $3(3.6 \%)$ \\
Others & $9(7.7 \%)$ \\
No detection & $7(10.7 \%)$ \\
\hline
\end{tabular}

Note: Including overlapping infection, the total number of bacteria detected was 84 . Abbreviations: ESBL, extended-spectrum beta-lactamase; sp, species; CNS, coagulase-negative staphylococci.

mean age of enrolled patients in our study was $74 \pm 15$ years and only $72 \%$ were females. Considering that older age and male sex increase the frequency of PFS, we believe that our findings were acceptable.

Previous reports did not include a control group, making it impossible to calculate the sensitivity and specificity. In our study, we included patients who had undergone renal biopsy as our control group, after acute pyelonephritis had been ruled out by the treating physician (given that active UTI is a contraindication for percutaneous renal biopsy). This allows us to calculate the sensitivity and specificity. In the control group, $67(21 \%)$ patients had positivity of WBC and/or nitrite in urinalysis; however, all of them had asymptomatic bacteriuria. There were $\sim 20 \%$ of patients with asymptomatic bacteriuria when the control group was compared to the whole population; it might be related to the fact that the average age was higher and there were more diabetic patients and immunosuppressant users.

Although hypoenhanced regions in the kidney may be characteristic of acute pyelonephritis, they are not specific. For example, acute renal infarction and immunoglobulin G4-related renal disease (IgG4RD) are accompanied by hypoenhanced regions in the kidney. ${ }^{15,16}$ In addition, acute renal infarction is also associated with fever, flank pain, and increased inflammatory markers, similar to acute pyelonephritis. However, elevated serum lactate dehydrogenase is found in almost all patients with acute renal infarction, and this may be useful in distinguishing acute renal infarction from acute pyelonephritis. These findings suggest that radiological imaging is not as useful as conventional examination in the diagnosis of acute pyelonephritis.
Previous studies have reported on the sensitivity and specificity of diagnosis of pyelonephritis. The sensitivity of urinalysis ( $>10 \mathrm{WBCs} /$ high power field $[\mathrm{HPF}]$ ) was reported to be $58 \%-82 \%$, while the specificity was $65 \%-86 \% .{ }^{17}$ The sensitivity of positive leukocyte esterase, nitrite, and leukocyte esterase plus nitrite tests was reported to be $74 \%-96 \%$, $35 \%-85 \%$, and $75 \%-84 \%$, respectively. In contrast, their specificity was $94 \%-98 \%, 92 \%-100 \%$, and $82 \%-89 \%$, respectively. ${ }^{18-21}$ The sensitivity of positive dipstick hematuria was $44 \%$, while its specificity was $88 \%{ }^{22}$ The sensitivity of finding of $>1$ bacterium per HPF by Gram stain (centrifuged urine) was $93 \%$, while the specificity was $95 \%{ }^{23}$ We demonstrated that the sensitivity of PFS in diagnosing acute pyelonephritis was $72 \%$, while the specificity was $60 \%$. This result shows that radiological imaging is not a particularly useful tool for the diagnosis of acute pyelonephritis.

Why then are CT scans often used for the diagnosis of acute pyelonephritis? We propose two reasons. One reason is that almost all the enrolled patients visited the emergency room. The emergency physician, in wanting to avoid missing life-threatening diseases, may order radiological imaging, including CT scan; this may thus contribute to an increased number of CT scans performed. Another reason is CT scan is easily available in Japan. Indeed, Japan is reported to have one of the highest numbers of CT machines in the world. ${ }^{24,25}$ However, studies have shown that excessive use of diagnostic imaging is not necessarily associated with improved medical care. ${ }^{26-28}$ In terms of cost, an abdominal CT scan $(\sim 10,000)$ is much more expensive than a urine dipstick test (1260). As shown earlier, CT scans are not particularly useful for the diagnosis of acute pyelonephritis. We believe that our findings provide a fair account of the usefulness of CT scan in diagnosing acute pyelonephritis.

We calculated the power to compare the ratios of the two groups. The ratio of the pyelonephritis group was 0.72 and that of the control group was 0.40 ; the alpha error was 0.05 , and even if the sample size was 89 , the detection power was as high as 0.99 .

Table 5 lists the various bacteria that were isolated from the positive urine cultures in our cohort; the causative organisms were similar to previous reports in Japan. ${ }^{29}$

We agree that our method of selection also created several limitations. Ideally, we would like to include healthy patients into our control group. Additionally, we did not obtain CT scan for patients without symptoms or disease. CT scans that were obtained were only for diagnosis or follow-up. Our selection bias also created a limitation. The control group was expected to have some form of renal disease. Primary 
renal disease such as glomerulonephritis, tubulointerstitial nephritis, and others may increase PFS, although the cause of renal dysfunction may have little effect on the frequency of PFS (data not shown). Our findings revealed that age, sex, and eGFR strongly affected PFS. Another limitation that we did not consider was previous history of pyelonephritis, which we hope will be addressed in future studies.

Another limitation of our study was the exclusion of children aged $<16$ years and pregnant women. However, this is because CT examination is not advisable for pregnant women and children. Admittedly, the occurrence of pyelonephritis is particularly high among pregnant women, with an estimated incidence of $0.5 \%-2 \% .{ }^{30}$ Therefore, it was unfortunate that we could not evaluate PFS in this population of patients.

\section{Conclusion}

Older age, male sex, and lower eGFR are associated with an increased occurrence of PFS. However, with respect to the diagnosis of acute pyelonephritis, the presence of PFS itself did not have particularly high sensitivity and/or specificity compared to other tests.

\section{Acknowledgment}

We thank Dr Takashi Hakamazuka (radiologist) for the analysis of images and his expert advice and Editage (www. editage.jp) for English language editing.

\section{Disclosure}

The authors report no conflicts of interest in this work.

\section{References}

1. Schrier RW. Infections of the upper urinary tract. Diseases of the Kidney and Urinary Tract. Philadelphia, PA: Lippincott, Williams \& Wilkins; 2001:847-869.

2. Leibovici L, Greenshtain S, Cohen O, Wysenbeek AJ. Toward improved empiric management of moderate to severe urinary tract infections. Arch Intern Med. 1992;152(12):2481-2486.

3. Craig WD, Wagner BJ, Travis MD. Pyelonephritis: radiologic-pathologic review. Radiographics. 2008;28(1):255-277.

4. Sandberg T, Stokland E, Brolin I, Lidin-Janson G, Svanborg Edén C. Selective use of excretory urography in women with acute pyelonephritis. J Urol. 1989;141(6):1290.

5. Kanel KT, Kroboth FJ, Schwentker FN, Lecky JW. The intravenous pyelogram in acute pyelonephritis. Arch Intern Med. 1988;148(10):2144.

6. Lim SK, Ng FC. Acute pyelonephritis and renal abscesses in adults correlating clinical parameters with radiological (computer tomography) severity. Ann Acad Med Singapore. 2011;40(9):407-413.
7. Stunell H, Buckley O, Feeney J, Geoghegan T, Browne RF, Torreggiani WC. Imaging of acute pyelonephritis in the adult. Eur Radiol. 2007;17(7):1820-1828

8. Yu TY, Kim HR, Hwang KE, Lee JM, Cho JH, Lee JH. Computed tomography findings associated with bacteremia in adult patients with a urinary tract infection. Eur J Clin Microbiol Infect Dis. 2016;35(11): $1883-1887$

9. Hammond NA, Nikolaidis P, Miller FH. Infectious and inflammatory diseases of the kidney. Radiol Clin NAm. 2012;50(2):259-270.

10. Chikamatsu Y, Matsuda K, Nagasawa T, et al. Quantification of bleeding volume using computed tomography and clinical complication after percutaneous renal biopsy. Clin Kidney J. 2017;10(1):9-15.

11. Austin PC. The performance of different propensity score methods for estimating marginal hazard ratios. Stat Med. 2013;32(16):2837-2849.

12. Rosenbaum PR, Rubin DB. The central role of the propensity score in observational studies for causal effects. Biometrika. 1983;70(1):41-55.

13. Kanda Y. Investigation of the freely-available easy-to-use software "EZR" (Easy R) for medical statistics. Bone Marrow Transplant. 2013;48(3):452-458.

14. Stevens PE, Levin A; Kidney Disease: Improving Global Outcomes Chronic Kidney Disease Guideline Development Work Group Members. Evaluation and management of chronic kidney disease: synopsis of the kidney disease: improving global outcomes 2012 clinical practice guideline. Ann Intern Med. 2013;158(11):825-830.

15. Nagasawa T, Matsuda K, Takeuchi $Y$, et al. A case series of acute renal infarction at a single center in Japan. Clin Exp Nephrol. 2016; 20(3):411-415.

16. Kawano M, Saeki T, Nakashima $\mathrm{H}$, et al. Proposal for diagnostic criteria for IgG4-related kidney disease. Clin Exp Nephrol. 2011;15(5):615-626.

17. Ferry S, Andersson SO, Burman LG, Westman G. Optimized urinary microscopy for assessment of bacteriuria in primary care. J Fam Pract. 1990;31(2):153-159.

18. Hooton TM, Stamm WE. Diagnosis and treatment of uncomplicated urinary tract infection. Infect Dis Clin North Am. 1997;11(3):551-581.

19. Pollock HM. Laboratory techniques for detection of urinary tract infection and assessment of value. Am J Med. 1983;75(1B):79-84.

20. Semeniuk H, Church D. Evaluation of the leukocyte esterase and nitrite urine dipstick screening tests for detection of bacteriuria in women with suspected uncomplicated urinary tract infections. J Clin Microbiol. 1999;37(9):3051-3052.

21. Pfaller MA, Koontz FP. Laboratory evaluation of leukocyte esterase and nitrite tests for the detection of bacteriuria. J Clin Microbiol. $1985 ; 21(5): 840-842$.

22. Blum RN, Wright RA. Detection of pyuria and bacteriuria in symptomatic ambulatory women. J Gen Intern Med. 1992;7(2):140-144.

23. Moyer VA, Elliott EJ, editors. Evidence Based Pediatrics and Child Health. 2nd ed. London: BMJ Books; 2004.

24. Berrington de González A, Darby S. Risk of cancer from diagnostic X-rays: estimates for the UK and 14 other countries. Lancet. 2004;363(9406):345-351.

25. OECD. Health at a Glance 2013 (Report). Paris: OECD; 2013:87.

26. Rehani B. Imaging overutilisation: is enough being done globally? Biomed Imaging Interv J. 2011;7(1):e6.

27. Emanuel EJ, Fuchs VR. The perfect storm of overutilization. JAMA. 2008;299(23):2789-2791.

28. Hurley R. Can doctors reduce harmful medical overuse worldwide? BMJ. 2014;3(349):g4289.

29. Kenzaka T, Kato K, Kitao A, et al. Hyperammonemia in urinary tract infections. PLoS One. 2015;10(8): 0136220.

30. Gilstrap LC 3rd, Ramin SM. Urinary tract infections during pregnancy. Obstet Gynecol Clin North Am. 2001;28(3):581. 


\section{Publish your work in this journal}

The International Journal of General Medicine is an international, peer-reviewed open-access journal that focuses on general and internal medicine, pathogenesis, epidemiology, diagnosis, monitoring and treatment protocols. The journal is characterized by the rapid reporting of reviews, original research and clinical studies across all disease areas.
The manuscript management system is completely online and includes a very quick and fair peer-review system, which is all easy to use. Visit $\mathrm{http}: / /$ www.dovepress.com/testimonials.php to read real quotes from published authors.

Submit your manuscript here: https://www.dovepress.com/international-journal-of-general-medicine-journal 